\title{
Efficacy and safety of eptinezumab in patients with chronic migraine
}

PROMISE-2

Richard B. Lipton, MD, Peter J. Goadsby, MD, PhD, Jeff Smith, MD, FRCP, Barbara A. Schaeffler, MBA, David M. Biondi, DO, Joe Hirman, PhD, Susan Pederson, BS, Brent Allan, DO, MPH, and Roger Cady, MD

Neurology ${ }^{\circledR}$ 2020;94:e1365-e1377. doi:10.1212/WNL.0000000000009169

\section{Abstract}

\section{Objective}

To evaluate the efficacy and safety of eptinezumab, a humanized anti-calcitonin gene-related peptide monoclonal antibody, in the preventive treatment of chronic migraine (CM).

\section{Methods}

The Prevention of Migraine via Intravenous ALD403 Safety and Efficacy-2 (PROMISE-2) study was a phase 3, multicenter, randomized, double-blind, placebo-controlled, parallel-group study. Adults with CM were randomly assigned to receive IV eptinezumab $100 \mathrm{mg}$, eptinezumab $300 \mathrm{mg}$, or placebo administered on day 0 and week 12 . The primary endpoint was change from baseline in mean monthly migraine days (MMDs) over weeks 1 to 12 .

\section{Results}

Among treated participants $(\mathrm{n}=1,072)$, baseline mean number of MMDs was $\approx 16.1$ across groups. Treatment with eptinezumab 100 and $300 \mathrm{mg}$ was associated with significant reductions in MMDs across weeks 1 to 12 compared with placebo (placebo -5.6, $100 \mathrm{mg}-7.7, p<0.0001$ vs placebo; $300 \mathrm{mg}-8.2, p<0.0001$ vs placebo). Treatment-emergent adverse events (TEAEs) were reported by $43.5 \%$ (100 mg), $52.0 \%$ (300 mg), and 46.7\% (placebo) of patients. Nasopharyngitis was the only TEAE reported for $>2 \%$ of eptinezumab-treated patients at an incidence of $>2 \%$ over placebo; it occurred in the $300 \mathrm{mg}$ eptinezumab arm (eptinezumab 9.4\%, placebo 6.0\%).

\section{Conclusion}

In patients with CM, eptinezumab 100 and $300 \mathrm{mg}$ was associated with a significant reduction in MMDs from the day after IV administration through week 12, was well tolerated, and demonstrated an acceptable safety profile.

\section{Classification of evidence}

This study provides Class I evidence that for patients with CM, a single dose of eptinezumab reduces MMDs over 12 weeks of treatment.

\author{
Correspondence \\ Dr. Lipton \\ richard.lipton@ \\ einsteinmed.org
}

\section{MORE ONLINE}

$\rightarrow$ Class of Evidence

Criteria for rating therapeutic and diagnostic studies

NPub.org/coe

\section{ClinicalTrials.gov identifier \\ NCT02974153.}




\section{Glossary}

ADA = anti-drug antibody; AE = adverse event; ANCOVA = analysis of covariance; $\mathbf{C G R P}=$ calcitonin gene-related peptide; $\mathbf{C M}=$ chronic migraine; eDiary = electronic diary; HIT-6 = 6-item Headache Impact Test; ICHD-3 = International Classification of Headache Disorders, 3rd edition; $\mathbf{M M D}=$ monthly migraine day; NAb = ADA with neutralizing potential; PROMISE = Prevention of Migraine via Intravenous ALD403 Safety and Efficacy; SAE = serious AE; TEAE = treatmentemergent AE.

Migraine is a common, disabling neurologic disorder ${ }^{1}$; its most burdensome form is chronic migraine (CM), clinically defined by the presence of headache on $\geq 15 \mathrm{~d} /$ mo for $\geq 3$ months, with $\geq 8 \mathrm{~d} / \mathrm{mo}$ of headaches linked to migraine. ${ }^{2}$ Relative to episodic migraine, CM is associated with significantly greater disability, higher rates of comorbidity, and increased direct and indirect costs. ${ }^{3}$

Reducing the burden of $\mathrm{CM}$ is an important goal of preventive treatment, although randomized trials in $\mathrm{CM}$ are a recent phenomenon. ${ }^{4-7}$ Currently, onabotulinumtoxinA and calcitonin gene-related peptide (CGRP)-targeted monoclonal antibodies are the only agents approved for the prevention of CM. In the United States, diagnosis and treatment rates for $\mathrm{CM}$ are low, and migraine preventive treatment is frequently discontinued prematurely. ${ }^{8-13}$

The Prevention of Migraine via Intravenous ALD403 Safety and Efficacy (PROMISE) phase 3 studies were randomized, double-blind, placebo-controlled trials designed to evaluate the efficacy, safety, and pharmacokinetics of repeat IV administration of the monoclonal antibody eptinezumab (ALD403) for migraine prevention in patients with episodic migraine (PROMISE-1) and CM (PROMISE-2). Eptinezumab is a monoclonal antibody that binds to the CGRP ligand. ${ }^{14,15}$ Blockade of the CGRP pathway is an established method in the acute and preventive treatment of migraine. ${ }^{16}$ This report presents the primary results of the PROMISE-2 study.

\section{Methods}

\section{Standard protocol approvals, registrations, and patient consents}

The study was approved by the independent ethics committee or institutional review board for each study site. All clinical work was conducted in compliance with current Good Clinical Practices as referenced in the International Conference on Harmonisation of Technical Requirements for Registration of Pharmaceuticals for Human Use guidelines, local regulatory requirements, and the principles of the Declaration of Helsinki. All patients enrolled in the study provided written informed consent before their participation. All clinical sites were required to sign a study-specific site blinding plan. This study is registered on ClinicalTrials. gov (NCT02974153).

\section{Study design and patients}

This phase 3, double-blind, randomized, placebo-controlled, parallel-group, efficacy and safety study was performed at 128 sites in 13 countries (United States, Spain, Ukraine, Russian Federation, United Kingdom, Republic of Georgia, Hungary, Italy, Slovakia, Germany, Czech Republic, Denmark, and Belgium) during the period of November 30, 2016, to April 20, 2018.

Adults 18 to 65 years of age (inclusive) with a diagnosis of migraine at or before 50 years of age were eligible for participation if they had a history of CM for $\geq 12$ months before screening, completed the headache electronic diary (eDiary) on $\geq 24$ of the 28 days after screening visit and before randomization (the screening period), and experienced $\geq 15$ to $\leq 26$ headache days and $\geq 8$ migraine days during the 28-day screening period. ${ }^{2}$ Patients taking prescription or over-thecounter medication for acute or preventive treatment of migraine were eligible only if the medications had been prescribed or recommended by a health care professional; migraine preventive medication use had to be stable for $\geq 3$ months before screening. Hormonal therapy (e.g., contraceptive, hormone replacement) was also permitted if it was stable and ongoing $\geq 3$ months before screening. Patients using barbiturates or prescription opioids $\leq 4 \mathrm{~d} /$ mo were eligible for participation if use was stable for $\geq 2$ months before screening, and this restriction was maintained through week 24 of the study. Other medications for the treatment of acute migraine such as triptans, nonsteroidal anti-inflammatory drugs, and simple analgesics were not restricted. Patients with CM and medication-overuse headache with the exception of the overuse of barbiturates or opioids were eligible for inclusion. The study steering committee wished to include patients using opioids and barbiturates because more than one-third of people in the United States who have migraine use these medications as acute prescription treatments. The study steering committee chose to limit opioid and barbiturate use because high use often makes the patient refractory to preventive treatments. In pursuit of a balance between generalizability and enrolling the target population, patients using opioids or barbiturates $\geq 5 \mathrm{~d} /$ mo were excluded.

Individuals were excluded from participation if they had a confounding pain disorder or clinically significant pain syndromes; uncontrolled or untreated psychiatric conditions; acute or active temporomandibular disorders; history or diagnosis of a headache or migraine disorders that did not meet the International Classification of Headache Disorders, 3rd edition (ICHD-3) 
beta version (2013) Section 1.3 criteria for CM; present or previous malignancies (except history of squamous or basal cell carcinoma with excision for cure or breast or cervical cancer $\geq 10$ years since diagnosis/treatment without evidence of recurrence); any active, progressive, or unstable cardiovascular, neurologic, or autoimmune disorder; newly diagnosed or uncontrolled hypertension (mild primary hypertension that was well controlled for $\geq 6$ months before screening was allowed); history or evidence of substance abuse or dependence; clinically significant abnormal ECG findings; a concurrent medical condition or laboratory abnormality during the screening period or before dosing on day 0; body mass index $\geq 39 \mathrm{~kg} / \mathrm{m}^{2}$; or recent or planned surgery requiring general anesthesia within 8 weeks before screening or during the duration of the study. Also excluded were patients who had received any experimental, unregistered therapy within 30 days or 5 plasma half-lives before screening or who had used any prohibited devices, neuromodulation, neurostimulation, or injectable therapy within 2 months before screening or during the screening period; botulinum toxin (any type) for migraine or for any other medical/ cosmetic reasons requiring injections within 4 months before screening or during the screening period; monoamine oxidase inhibitors, ketamine, methylergonovine, or nimesulide within 3 months before screening or during the screening period; any monoclonal antibody treatment within 6 months of screening; or eptinezumab or any monoclonal antibody targeting the CGRP pathway. Women who were pregnant, breastfeeding, or planning to become pregnant during the study were excluded from participation, as were patients participating in any other clinical study or who were positive for HIV, hepatitis B surface antigen, or hepatitis $\mathrm{C}$.

Women of childbearing potential and men with partners of childbearing potential agreed to use adequate contraception throughout study participation and for $\geq 6$ months after the last dose of study drug.

\section{Study procedures}

Patients used an eDiary to document headaches and migraines for 4 weeks after the screening visit to confirm eligibility criteria and to establish baseline values. Eligible patients were then randomly assigned to receive eptinezumab $100 \mathrm{mg}$, eptinezumab $300 \mathrm{mg}$, or placebo in a 1:1:1 ratio. Randomization was stratified by the number of migraine days recorded during the screening period ( $<17 \mathrm{vs} \geq 17$ days) and preventive medication use during the 3 months before screening (use vs no use). The total duration of the study was 32 weeks, with 10 scheduled visits (screening, day 0 , and weeks $2,4,8,12,16,20,24$, and 32). After the last patient completed the week 12 visit, the analysis of the primary endpoint was performed.

Patients received up to 2 treatments of eptinezumab or placebo (administered IV on day 0 and at week 12). Assignment was concealed. Investigational product (either eptinezumab $100 \mathrm{mg}$ or placebo) was shipped frozen on dry ice as a singleuse, preservative-free solution in a tamper-proof 2-mL type I glass vial. Each individual vial was labeled with protocol- specific information to meet International Conference on Harmonisation of Technical Requirements for Registration of Pharmaceuticals for Human Use and country-specific requirements. On randomization, study sites were provided with enough appropriate investigational product for 1 patient to be dosed, with subsequent treatment packs shipped automatically before the next scheduled treatment visit. Investigational treatments were reconstituted in a total volume of $100 \mathrm{~mL} 0.9 \%$ saline and administered over a period of 30 minutes (plus an additional 15 minutes, per protocol, if required) by the blinded investigator. Patients were monitored for $\geq 2$ hours after treatment. All research participants, clinicians, and research personnel were blinded and remained blinded throughout the duration of the clinical trial.

\section{Outcome measures}

Patients completed a daily eDiary from the time of screening through week 24; this included a daily evening report (completed regardless of whether the patient had a headache) and a headache report, which was event based (i.e., per headache). In addition to capturing headache episodes and migraine attacks, the daily eDiary captured acute medication use (including type). A migraine day was defined using information from the eDiary and followed the definition of a CM outlined in ICHD-3. Specifically, a migraine day had to meet 3 criteria: (1): lasted $\geq 4$ hours or lasted 30 minutes to 4 hours and believed by the patient to be a migraine that was relieved by acute medication; (2) had $\geq 2$ of the following: unilateral location, pulsating quality, moderate or severe pain intensity, and aggravation by or causing avoidance of routine physical activity; and (3) had $\geq 1$ of the following: nausea and/or vomiting or photophobia and phonophobia.

Monthly results (e.g., monthly migraine days [MMDs]) were based on the results of the 4-week intervals. If the headache diary was completed for $\geq 21$ days of a 4-week interval, the observed frequency was normalized to 28 days. If the diary was completed for $<21$ days of a 4-week interval, the results were a weighted function of the observed data for the current interval and the results from the previous interval, with the weight proportional to how many days the diary had been completed.

The primary efficacy endpoint was the change from baseline in MMDs over weeks 1 to 12 , assessed with eDiary data. eDiary data also were used for evaluating several of the key secondary efficacy endpoints: $\geq 75 \%$ migraine responder rate over weeks 1 to $4, \geq 75 \%$ migraine responder rate over weeks 1 to $12, \geq 50 \%$ migraine responder rate over weeks 1 to 12 , percentage of patients with a migraine on the day after dosing, change from baseline in daily migraine prevalence from baseline to week 4 , and acute migraine medication use during weeks 1 to 12 .

During the scheduled visits, patients completed several patient-reported outcome measures, including the 6-item Headache Impact Test (HIT-6), the 36-item Short-Form Health Survey (version 2.0), the EuroQol 5-Dimensions 5-Levels, and the Patient Global Impression of Change. The 
Most Bothersome Symptom was evaluated as a tertiary endpoint and is not reported here.

The HIT- $6^{17,18}$ version 1.0, an a control endpoint in this study for the $300 \mathrm{mg}$ treatment group, was administered at screening, day 0 , and at week $4,12,16,24$, and 32 visits to measure the impact of headaches on the ability to function normally in daily life. Patients rated the HIT-6 items using a Likert-type scale anchored by "never" and "always" and including the following response scores: never $=6$, rarely $=8$, sometimes $=10$, very often $=11$, and always $=13$. Response scores were summed to produce a total score. Scores $\geq 60$ were indicative of severe life impact, 56 to 59 of substantial life impact, 50 to 55 of some life impact, and $\leq 49$ of little to no life impact.

Safety was assessed throughout the study via adverse event (AE) monitoring, clinical laboratory tests, vital sign measurements, physical examinations, 12-lead ECGs, the ColumbiaSuicide Severity Rating Scale, ${ }^{19}$ and concomitant medication use. AEs of special interest also were monitored and included AEs associated with the Columbia-Suicide Severity Rating Scale score, cardiovascular AEs, hepatic AEs, and those associated with study drug administration, including hypersensitivity and anaphylaxis AEs.

Blood samples for immunogenicity analyses were collected on day 0 and at week 2, 4, 8, 12, 24, and 32, including monitoring development of anti-eptinezumab antibodies and assaying for neutralizing potential.

\section{Statistical methods}

A total of 350 patients per group provided at least $90 \%$ power for the primary endpoint for each comparison, assuming a treatment effect of $\geq 1$ day and a common SD of $\leq 4$ days. These sample size calculations were performed with PASS $2008^{20}$ and were based on a $t$ test that approximated the analysis of covariance (ANCOVA) used for the primary endpoint. ${ }^{20}$

All patients who received study medication were included in the safety and efficacy populations. For the safety analyses, patient results were summarized within the group representing the treatment they received; if they received 2 different doses, they were summarized in the treatment arm of the highest dose received. For the efficacy population, patients' results were summarized within the treatment group to which they were randomly assigned.

A serial procedure was used to account for multiplicity associated with $>1$ dose level and for multiple endpoints (figure 1). Statistical testing was conducted to maintain a study-wide 2 -sided 5\% a level. The procedure first evaluated the $300 \mathrm{mg}$ vs placebo comparison for the primary endpoint. If this was significant, testing continued to the first subset of key secondary endpoints for $300 \mathrm{mg}$. If a significant difference was detected for all tests within this series, the procedure then moved on to the second subset of key secondary endpoints for $300 \mathrm{mg}$. The procedure then moved on to the $100 \mathrm{mg}$ group for the primary endpoint and subsequently the key secondary endpoint groups for $100 \mathrm{mg}$. If these endpoints were significant, the procedure moved to the final key secondary endpoints for $300 \mathrm{mg}$. Within each endpoint group, the Holm procedure ${ }^{21}$ was used.

The primary efficacy endpoint was the change from baseline in MMDs (weeks 1-12) in an ANCOVA model. Key secondary efficacy endpoints were $\geq 75 \%$ migraine responder rates during weeks 1 to 4 and 1 to $12, \geq 50 \%$ migraine responder rates during weeks 1 to 12 , percentage of patients with a migraine on the day after dosing, reduction in average daily percentage of patients with migraine from baseline to week 4, change in HIT-6 total score from baseline to week 12 (300 mg dose only), and acute migraine medication use from baseline to week 12 (300 mg dose only). A $\geq 50 \%$ or $\geq 75 \%$ migraine responder was defined as a patient who achieved $\geq 50 \%$ reduction or $\geq 75 \%$ reduction in MMDs from baseline, respectively. These reductions were evaluated by comparing the baseline frequency of migraine days to the migraine frequency in 4-week intervals. Results from these 4-week intervals will be combined to produce 12 -week responder endpoints. The week 1 to 4,5 to 8 , and 9 to 12 changes from baseline measures were averaged, and the average value was compared to baseline. A percent change from baseline was determined, which was used to determine the responder status.

For the key secondary endpoints (migraine responder rates and percentage of patients with a migraine on the day after dosing), testing was based on Cochran-Mantel-Haenszel/extended Cochran-Mantel-Haenszel tests. The tests were stratified by the randomization stratification factor. For the HIT-6 and Acute Migraine Medication endpoints, an ANCOVA model similar to the one used for the primary endpoint was used for testing. Exploratory and safety endpoints were summarized with descriptive statistics. All analyses were conducted with SAS software (SAS Institute, Inc, Cary, NC) version 9.2 or higher. Appendix 2 available from Dryad (doi.org/10.5061/ dryad.ck84q23) provides additional information on the statistical analysis.

\section{Data availability}

The data reported are part of an ongoing, global sponsor-led clinical development and registration program. Deidentified participant data are not available for legal and ethical reasons.

\section{Results}

A total of 1,121 patients were randomly assigned; 1,072 received treatment and were included in the safety and full analysis populations (figure 2). Reasons that patients were randomized but not treated included withdrawal by the patient (19 patients), loss to follow-up (3 patients), and other (e.g., randomization errors and protocol violations 27 patients). Overall, 1,049 patients (93.6\%) remained in the study until week 12 , the end of the primary efficacy period. A total of 1,000 patients (89.2\%) attended the week 24 visit, and 873 patients $(77.9 \%)$ attended the week 32 visit. 
Figure 1 Decision rule for dose levels (primary and key secondary endpoints)

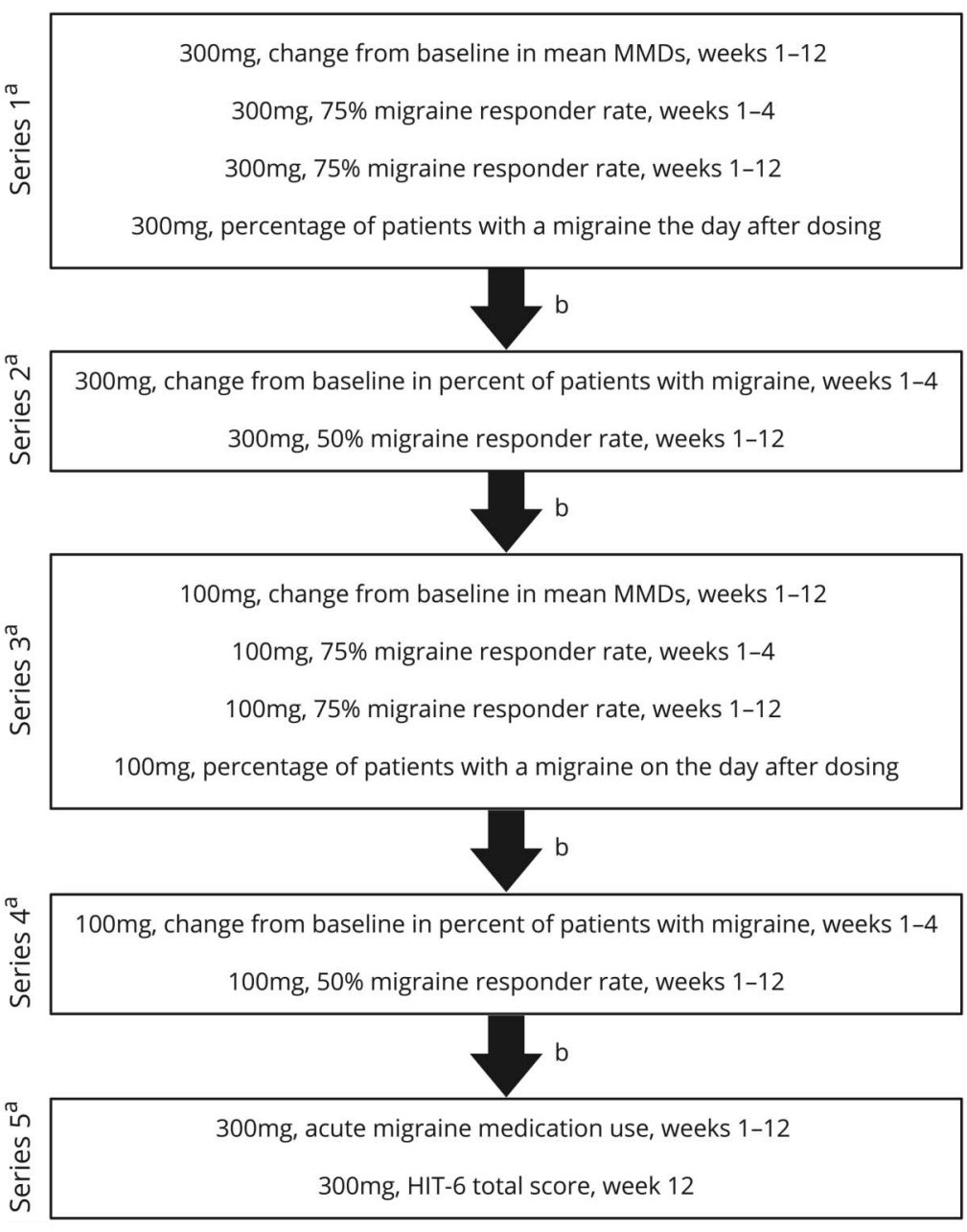

HIT-6 = Headache Impact Test-6; MMD = monthly migraine

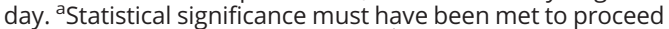
to the next step with each series. ${ }^{b}$ To proceed to the next series, all tests in the previous series must have shown a statistically significant difference from placebo.
Demographic and baseline clinical characteristics are summarized in table 1 . The mean age was 40.5 years; $65.7 \%$ of patients were $>35$ years of age. The majority of patients were female $(88.2 \%)$, white $(91.0 \%)$, and not Hispanic or Latino (92.0\%). Migraine history was well balanced across treatment groups. The mean age at migraine diagnosis was 22.5 years, and the mean duration of migraine diagnosis was 18.1 years. A total of 479 patients (44.7\%) used concomitant prophylactic medication. A total of 431 patients $(40.2 \%)$ had a diagnosis of medication-overuse headache at baseline. Diagnosis was made at screening by the study investigator using ICHD-3 beta criteria for medication-overuse headache and was confirmed by analyses of acute medication use over the baseline period.

Nearly all patients (99.7\%) reported using $\geq 1$ concomitant medications during the study; concomitant medication use was well balanced across treatment groups at the drug class level. The most frequently reported concomitant medications were nervous system medications (93.9\%), musculoskeletal system medications $(57.8 \%)$, alimentary tract and metabolism medications (33.5\%), respiratory system medications
(29.2\%), genitourinary system and sex hormone medications (26.1\%), and cardiovascular system medications (22.9\%).

\section{Efficacy findings}

Because the largest $p$ value within the $\alpha$-controlled primary and key secondary endpoints was 0.0001 based on the decision rule outlined in figure 1, the results for the primary efficacy endpoint and all key secondary endpoints for both eptinezumab dose groups were statistically significant (table 2).

\section{Primary efficacy endpoint}

Both 100 and $300 \mathrm{mg}$ of eptinezumab demonstrated statistically significant reductions in MMDs during weeks 1 to 12 ( $p$ < 0.0001; figure 3 and table 2). MMDs decreased from 16.1 to 8.5 days in the eptinezumab $100 \mathrm{mg}$ group, from 16.1 to 7.9 days in the eptinezumab $300 \mathrm{mg}$ group, and from 16.2 to 10.5 days in the placebo group. Relative to placebo, eptinezumab reduced mean (95\% confidence interval) MMDs from baseline (during the 28-day screening period) by $-2.0(-2.9$ to -1.2$)$ days for the $100 \mathrm{mg}$ dose and $-2.6(-3.4$ to -1.7$)$ days for the $300 \mathrm{mg}$ dose. A post hoc analysis of the primary efficacy endpoint in patients with no barbiturate or opioid use at any time during 


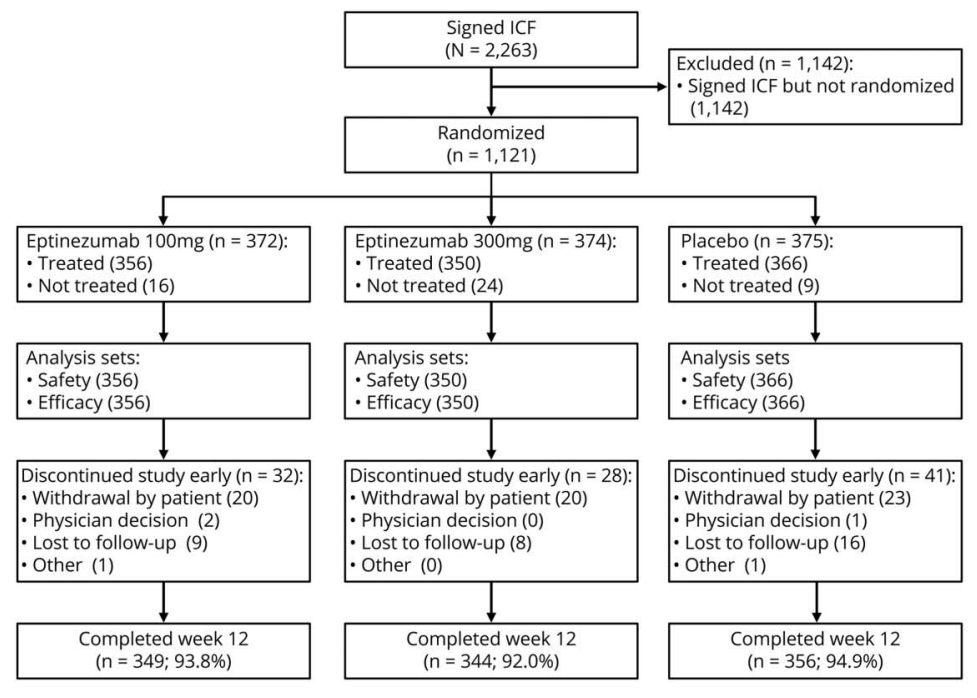

$\mathrm{ICF}=$ informed consent form.

the clinical trial identified a similar reduction in mean MMDs from baseline compared to placebo $(100 \mathrm{mg}-2.3[-3.2$ to $-1.4], p<0.0001 ; 300 \mathrm{mg}-2.8[-3.7$ to -1.9$], p<0.0001)$.

\section{Key secondary efficacy endpoints}

The $\geq 75 \%$ migraine responder rates (weeks $1-4$ and 1-12) and $\geq 50 \%$ migraine responder rates (weeks 1-12) are summarized in figure 4 and table 2. Patients in both eptinezumab dose groups were more likely to achieve $\geq 75 \%$ migraine response during weeks 1 to 4 than were patients in the placebo group (common odds ratios [95\% confidence interval] 2.4 [1.7-3.5] for eptinezumab $100 \mathrm{mg}$ and 3.2 [2.2-4.6] for eptinezumab $300 \mathrm{mg}$ ). They also were more likely to achieve $\geq 75 \%$ migraine response during weeks $1-12$ (common odds ratios [95\% confidence interval] 2.1 [1.4-3.0] for eptinezumab $100 \mathrm{mg}$ and 2.8 [1.9-4.0] for eptinezumab $300 \mathrm{mg}$ ) and $\geq 50 \%$ migraine response during weeks 1 to 12 (common odds ratios [95\% confidence interval] 2.1 [1.6-2.8] for eptinezumab $100 \mathrm{mg}$ and 2.4 [1.8-3.3] for eptinezumab $300 \mathrm{mg}$ ).

The migraine preventive effect of eptinezumab was statistically significant for the 100 and $300 \mathrm{mg}$ doses of eptinezumab vs placebo after the first day after dosing (figure 4). During the 28day screening period, the average daily percentage of patients with a migraine was $58 \%$. The percentage of patients with a migraine on the day after dosing was $28.6 \%$ in the eptinezumab $100 \mathrm{mg}$ group and $27.8 \%$ in the eptinezumab $300 \mathrm{mg}$ group vs $42.3 \%$ in the placebo group (both $p<0.0001$ vs placebo). Estimated mean changes in the average daily percentage of patients with a migraine from baseline over weeks $1,2,3$, and 4 were $-27.1 \%$ in the eptinezumab $100 \mathrm{mg}$ group (mean difference [95\% confidence interval] from placebo $-8.3 \%[-11.48$ to $-5.05], p<0.0001]),-29.8 \%$ in the eptinezumab $300 \mathrm{mg}$ group (mean difference from placebo $-11.0 \%$ [ -14.2 to -7.8$], p<$ 0.0001 ), and $-18.8 \%$ in the placebo group. Furthermore, the average daily percentage of patients with migraine was lower in the eptinezumab groups than in the placebo group during each of the first 4 weeks after dosing (figure 4).

Most patients had HIT- 6 total scores in the severe range at baseline (eptinezumab $100 \mathrm{mg} 89.6 \%$, eptinezumab $300 \mathrm{mg}$ $88.6 \%$, and placebo $87.4 \%$ ). The mean HIT-6 scores at baseline were 65.0 (eptinezumab $100 \mathrm{mg}$ ), 65.1 (eptinezumab $300 \mathrm{mg}$ ), and 64.8 (placebo). By week 12, the percentage of patients with HIT-6 scores in the severe range had been reduced to $51.4 \%$ in the eptinezumab $100 \mathrm{mg}$ treatment group, $42.9 \%$ in the eptinezumab $300 \mathrm{mg}$ treatment group, and $60.1 \%$ in the placebo group. Patients in the eptinezumab $300 \mathrm{mg}$ group demonstrated a statistically significant improvement on the HIT-6 at week 12 , with an estimated mean difference from placebo (95\% confidence interval $)$ of $-2.9(-3.9$ to $-1.8, p<0.0001)$. The estimated mean difference from placebo for the $100 \mathrm{mg}$ treatment group (a prespecified endpoint) was -1.7 and was nominally significant $(p=0.001)$, although this comparison was not formally included within the prespecified testing algorithm.

Both eptinezumab dose groups reduced acute medication days from baseline to week 12 (100 mg -3.3 days, $300 \mathrm{mg}$ -3.5 days). The reduction in the $100 \mathrm{mg}$ group was greater than that in the placebo group $(-1.2[-1.7$ to -0.6$], p<$ $0.0001)$ but was not formally tested. The reduction in the eptinezumab $300 \mathrm{mg}$ group was significantly greater than that in the placebo group (mean difference from placebo $[95 \%$ confidence interval $]-1.4[-1.9$ to -0.9$], p<0.0001)$.

\section{Safety and tolerability}

A total of 1,072 patients received $\geq 1$ doses of study medication. The majority of patients received both doses of eptinezumab $(100 \mathrm{mg} \mathrm{n}=340$ [95.5\%], $300 \mathrm{mg} \mathrm{n}=338$ [96.6\%]) or placebo $(\mathrm{n}=342[93.4 \%])$. 
Table 1 Demographics and baseline clinical characteristics (safety population)

\begin{tabular}{|c|c|c|c|c|}
\hline & $\begin{array}{l}\text { Eptinezumab } 100 \mathrm{mg} \\
(\mathrm{n}=356)\end{array}$ & $\begin{array}{l}\text { Eptinezumab } 300 \mathrm{mg} \\
(\mathrm{n}=350)\end{array}$ & $\begin{array}{l}\text { Placebo } \\
(\mathrm{n}=366)\end{array}$ & $\begin{array}{l}\text { Total population } \\
(n=1,072)\end{array}$ \\
\hline Mean (SD) age, y & $41.0(11.7)$ & $41.0(10.4)$ & $39.6(11.3$ & $40.5(11.2)$ \\
\hline \multicolumn{5}{|l|}{ Sex, n (\%) } \\
\hline Male & $49(13.8)$ & $36(10.3)$ & $41(11.2)$ & $126(11.8)$ \\
\hline Female & $307(86.2)$ & $314(89.7)$ & $325(88.8)$ & $946(88.2)$ \\
\hline \multicolumn{5}{|l|}{ Ethnicity, n (\%) } \\
\hline Hispanic or Latino & $33(9.3)$ & $18(5.1)$ & $35(9.6)$ & $86(8.0)$ \\
\hline Not Hispanic or Latino & $323(90.7)$ & 332 (94.9) & $331(90.4)$ & $986(92.0)$ \\
\hline \multicolumn{5}{|l|}{ Race, n (\%) } \\
\hline White & $332(93.3)$ & $322(92.0)$ & $321(87.7)$ & $975(91.0)$ \\
\hline Black or African American & $21(5.9)$ & $23(6.6)$ & $38(10.4)$ & $82(7.6)$ \\
\hline Asian & $1(<1)$ & $1(<1)$ & $1(<1)$ & $3(<1)$ \\
\hline American Indian or Alaska Native & $1(<1)$ & $1(<1)$ & $1(<1)$ & $3(<1)$ \\
\hline Native Hawaiian or other Pacific Islander & 0 & $1(<1)$ & 0 & $1(<1)$ \\
\hline Multiple races & $1(<1)$ & $2(<1)$ & $4(1.1)$ & $7(<1)$ \\
\hline Other & 0 & 0 & $1(<1)$ & $1(<1)$ \\
\hline Mean (SD) weight, kg & $73.3(15.7)$ & $72.7(15.3)$ & $74.9(16.3)$ & $73.6(15.8)$ \\
\hline Mean (SD) height, cm & $166.2(8.2)$ & $166.1(7.9)$ & $166.3(7.9)$ & $166.2(8.0)$ \\
\hline Mean (SD) BMI, kg/m² & $26.4(5.0)$ & $26.2(5.0)$ & $27.0(5.6)$ & $26.6(5.2)$ \\
\hline Mean (SD) age at migraine diagnosis, y & $22.8(10.6)$ & $22.0(9.3)$ & $22.6(10.0)$ & $22.5(10.0)$ \\
\hline $\begin{array}{l}\text { Mean (SD) duration of migraine } \\
\text { diagnosis at baseline, } y\end{array}$ & $18.3(12.2)$ & $19.0(11.5)$ & $17.0(11.6)$ & $18.1(11.8)$ \\
\hline Mean (SD) duration of $\mathrm{CM}$ at baseline, $y$ & $11.6(11.7)$ & $12.4(11.2)$ & $11.6(10.9)$ & $11.8(11.2)$ \\
\hline Mean (SD) No. of headache days ${ }^{a}$ & $20.4(3.1)$ & $20.4(3.2)$ & $20.6(2.99)$ & $20.5(3.1)$ \\
\hline Mean (SD) No. of migraine days ${ }^{a}$ & $16.1(4.6)$ & $16.1(4.8)$ & $16.2(4.6)$ & $16.1(4.6)$ \\
\hline Medication-overuse headache diagnosis, n (\%) & $139(39.0)$ & $147(42.0)$ & 145 (39.6) & $431(40.2)$ \\
\hline \multicolumn{5}{|l|}{ Baseline triptan use, n (\%) } \\
\hline$\geq 33 \%$ of days & $124(34.8)$ & $125(35.7)$ & $116(31.7)$ & $365(34.0)$ \\
\hline$<33 \%$ of days & $231(64.9)$ & $225(64.3)$ & $250(68.3)$ & $706(65.9)$ \\
\hline Use of opioids or barbiturates at any time, $n$ (\%) & 46 (12.9) & $39(11.1)$ & $48(13.1)$ & $133(12.4)$ \\
\hline
\end{tabular}

Abbreviations: $\mathrm{BMI}=$ body mass index; $\mathrm{CM}=$ chronic migraine.

a Full analysis population; mean electronic diary-reported migraine and headache characteristics during the 28-day screening period.

\section{Adverse events}

Overall, 508 patients (47.4\%) experienced $\geq 1$ treatmentemergent AEs (TEAEs). The incidence of TEAEs was generally balanced among treatment groups (table 3 ).

A total of 122 patients (11.4\%) had $\geq 1$ TEAEs considered by the investigator to be related to the study drug: 93 patients (13.2\%) in the eptinezumab groups and 29 patients $(7.9 \%)$ in the placebo group. The most frequently reported study drug-related TEAEs were fatigue (eptinezumab $\mathrm{n}=13[1.8 \%]$, placebo $\mathrm{n}=3[<1 \%]$ ) and nausea (eptinezumab $\mathrm{n}=11[1.6 \%]$, placebo $\mathrm{n}=1[<1 \%])$. The remaining study drug-related TEAEs occurred in $<1 \%$ of patients.

No deaths were reported in this study. A total of 10 patients $(<1 \%)$ experienced a serious TEAE ( 7 patients who received 
Table 2 Efficacy outcomes (efficacy population)

Eptinezumab $100 \mathrm{mg}$

$(n=356)$
Eptinezumab $300 \mathrm{mg}$

$(n=350)$
Placebo

(n = 366)

Mean MMDs, weeks 1-12

Actual

$$
\text { Mean }
$$

Change from baseline

$$
\text { Mean }
$$

Difference from placebo $(95 \% \mathrm{CI})$

$p$ Value vs placebo

$-2.0(-2.9$ to -1.2$)$

$<0.0001$
$-8.2$

$-2.6(-3.4$ to -1.7$)$

$-5.6$

$<0.0001$

Mean headache days, weeks 1-12

Actual

$$
\text { Mean }
$$

12.2

11.7

14.1

Change from baseline

Mean -8.2

Difference from placebo $(95 \% \mathrm{CI})$

$-8.2$

$-8.8$

$-1.7(-2.6$ to -0.9$)$

$-2.3(-3.2$ to -1.4$)$

Percentage of patients with migraine, day 1

Actual

$$
\text { Mean }
$$

28.6

27.8

$p$ Value vs placebo

$<0.0001$

$<0.0001$

Average daily migraine prevalence,

weeks 1-4, \%

\section{Actual}

$$
\text { Mean }
$$

Change from baseline

$$
\text { Mean }
$$

$-27.1$

$-29.8$

Difference from placebo $(95 \% \mathrm{CI})$

$p$ Value vs placebo

\begin{tabular}{|c|c|c|c|}
\hline Patients, n (\%) & $110(30.9)$ & 129 (36.9) & $57(15.6)$ \\
\hline Difference from placebo $(95 \% \mathrm{CI})$ & 15.3 (9.3 to 21.4$)$ & $21.3(15.0$ to 27.6$)$ & \\
\hline$p$ Value vs placebo & $<0.0001$ & $<0.0001$ & \\
\hline Odds ratio vs placebo & 2.4 & 3.2 & \\
\hline \multicolumn{4}{|c|}{ 75\% Migraine responder rate, weeks 1-12 } \\
\hline Patients, n (\%) & $95(26.7)$ & $116(33.1)$ & $55(15.0)$ \\
\hline Difference from placebo $(95 \% \mathrm{CI})$ & 11.7 (5.8 to 17.5$)$ & $18.1(12.0$ to 24.3$)$ & \\
\hline$p$ Value vs placebo & 0.0001 & $<0.0001$ & \\
\hline Odds ratio vs placebo & 2.1 & 2.8 & \\
\hline \multicolumn{4}{|c|}{$50 \%$ Migraine responder rate, weeks 1-12 } \\
\hline Patients, n (\%) & $205(57.6)$ & $215(61.4)$ & $144(39.3)$ \\
\hline Difference from placebo $(95 \% \mathrm{CI})$ & $18.2(11.1$ to 25.4$)$ & 22.1 (14.9 to 29.2$)$ & \\
\hline
\end{tabular}

$-8.3(-11.5$ to -5.0$)$

$-11.0(-14.2$ to -7.8$)$

$<0.0001$

$<0.0001$

75\% Migraine responder rate, weeks 1-4 
Table 2 Efficacy outcomes (efficacy population) (continued)

\begin{tabular}{|c|c|c|c|}
\hline & $\begin{array}{l}\text { Eptinezumab } 100 \mathrm{mg} \\
(\mathrm{n}=356)\end{array}$ & $\begin{array}{l}\text { Eptinezumab } 300 \mathrm{mg} \\
(\mathrm{n}=350)\end{array}$ & $\begin{array}{l}\text { Placebo } \\
(n=366)\end{array}$ \\
\hline$p$ Value vs placebo & $<0.0001$ & $<0.0001$ & \\
\hline Odds ratio vs placebo & 2.1 & 2.4 & \\
\hline \multicolumn{4}{|l|}{ Mean acute medication days, weeks 1-12 } \\
\hline \multicolumn{4}{|l|}{ Actual } \\
\hline Mean & 3.3 & 3.2 & 4.3 \\
\hline \multicolumn{4}{|l|}{ Change from baseline } \\
\hline Mean & -3.3 & -3.5 & -1.9 \\
\hline Difference from placebo $(95 \% \mathrm{Cl})$ & $-1.2(-1.7$ to -0.6$)$ & $-1.4(-1.9$ to -0.9$)$ & \\
\hline$p$ Value vs placebo & $<0.0001$ & $<0.0001$ & \\
\hline \multicolumn{4}{|l|}{ HIT-6 score, week 4} \\
\hline \multicolumn{4}{|l|}{ Actual } \\
\hline Mean & 58.0 & 56.4 & 60.3 \\
\hline \multicolumn{4}{|l|}{ Change from baseline } \\
\hline Mean & -6.9 & -8.6 & -4.6 \\
\hline Difference from placebo $(95 \% \mathrm{Cl})$ & $-2.3(-3.4$ to -1.2$)$ & $-4.0(-5.1$ to -2.8$)$ & \\
\hline \multicolumn{4}{|l|}{ HIT-6 score, week 12} \\
\hline \multicolumn{4}{|l|}{ Actual } \\
\hline Mean & 58.8 & 57.6 & 60.5 \\
\hline \multicolumn{4}{|l|}{ Change from baseline } \\
\hline Mean & -6.2 & -7.3 & -4.5 \\
\hline Difference from placebo $(95 \% \mathrm{Cl})$ & $-1.7(-2.8$ to -0.7$)$ & $-2.9(-3.9$ to -1.8$)$ & \\
\hline$p$ Value vs placebo & 0.0010 & $<0.0001$ & \\
\hline
\end{tabular}

Abbreviations: $\mathrm{Cl}=$ confidence interval; HIT-6 = Headache Impact Test- 6 ; MMD = monthly migraine day.

eptinezumab and 3 who received placebo). The most frequently reported serious TEAEs were in the system organ class of nervous system disorders (eptinezumab $n=4[<1 \%]$, placebo $\mathrm{n}=1[<1 \%])$; injury, poisoning, and procedural complications (eptinezumab $\mathrm{n}=2[<1 \%]$, placebo $\mathrm{n}=0$ ); and psychiatric disorders (eptinezumab $\mathrm{n}=2[<1 \%]$, placebo $\mathrm{n}=0)$. One serious AE (SAE) was assessed as related to the study drug by both the investigator and the sponsor. This was a single episode of worsening migrainous visual phenomena in a patient with a medical history of migraine with visual aura, considered part of the disease under study, which occurred 126 days after the second dose of eptinezumab $300 \mathrm{mg}$ and lasted 4 days. No patients had life-threatening SAEs.

A total of 13 patients (1.2\%) experienced a TEAE that led to study drug withdrawal: $3(<1 \%)$ in the eptinezumab $100 \mathrm{mg}$ group, $8(2.3 \%)$ in the eptinezumab $300 \mathrm{mg}$ group, and $2(<1 \%)$ in the placebo group. A total of 6 patients (1.7\%) who received eptinezumab $300 \mathrm{mg}$ had the study drug withdrawn due to hypersensitivity. Three of these events occurred after the first dose and 3 after the second dose. All were mild to moderate in severity, were considered related to the study drug, and occurred on the treatment day. Five of the 6 events resolved on the same day as onset ( 1 resolved within 2 days). The occurrence of individual symptoms or symptom constellations coded as the preferred term of hypersensitivity was based on the sponsor's established framework for evaluating treatment day events. The clinical presentation of these events was most commonly reported as nonspecific allergy-type reactions. Hypersensitivity symptoms (i.e., immunoglobulin $\mathrm{E}$ mediated) such as hives, angioedema, and documented respiratory manifestations were rare.

One patient who received eptinezumab $300 \mathrm{mg}$ experienced an SAE of seizure that required hospitalization and led to 
Figure 3 Primary endpoint: Change from baseline to week 12 in mean monthly migraine days (full analysis population)

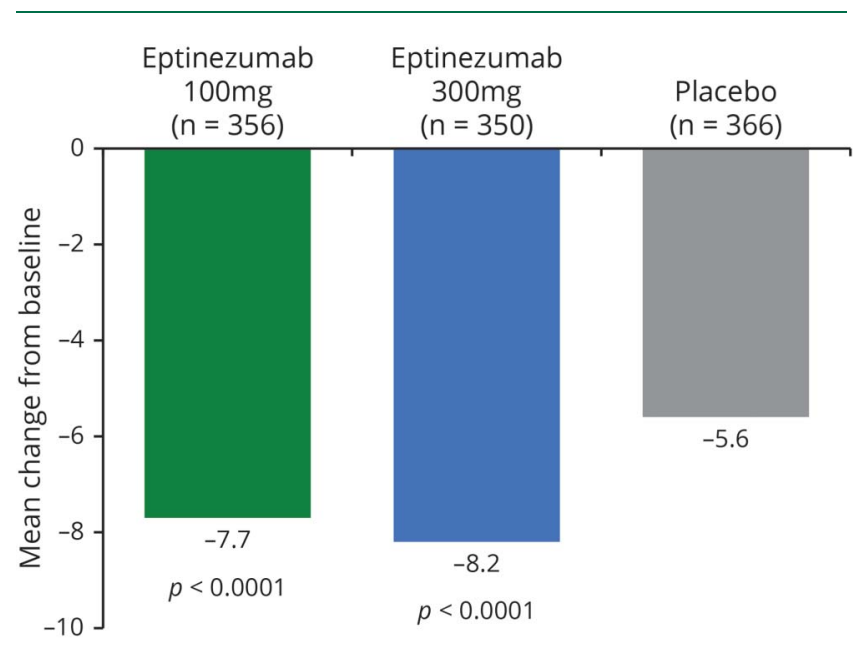

study drug withdrawal. This SAE was assessed by the investigator as not related to the study drug and resolved on the same day as the hospitalization. All TEAEs of special interest occurred in $<1 \%$ of the total eptinezumab and placebo groups.

\section{Anti-drug antibodies}

The incidence of anti-eptinezumab antibodies was maximal at 24 weeks (eptinezumab $100 \mathrm{mg} 56$ of 326 patients, eptinezumab $300 \mathrm{mg} 56$ of 329 patients) and then declined despite continuation of dosing (week 32: eptinezumab $100 \mathrm{mg} 37$ of 325, eptinezumab $300 \mathrm{mg} 29$ of 321 patients). There was no clear dose-response trend in the number of patients with antidrug antibody (ADA)-positive results. Among ADA-positive patients, 26 in the eptinezumab $100 \mathrm{mg}$ group and 19 in the eptinezumab $300 \mathrm{mg}$ group had $\mathrm{ADAs}$ with neutralizing potential (NAbs). The incidence of NAbs generally increased over time from week 2 to 24 (except at week 4). Of the 112 ADA-positive patients at week 24, NAb-positive status was reported in 24 patients (21.4\%). Formation of $\mathrm{ADAs}$ with or without neutralizing potential did not affect efficacy (change in MMDs over weeks 1-12 for ADA-positive vs ADAnegative patients: eptinezumab $100 \mathrm{mg}-6.1 \mathrm{vs}-8.1$, eptinezumab $300 \mathrm{mg}-9.3$ vs -8.1 , respectively). Similarly, the results showed no evidence of an impact from the development of $\mathrm{ADA}$, including NAbs, on the safety profile of eptinezumab.

\section{Discussion}

PROMISE-2 demonstrated that both the 100 and $300 \mathrm{mg}$ doses of eptinezumab resulted in significant reductions in the primary endpoint, MMDs, over weeks 1 to 12 after the first IV administration. The $\geq 75 \%$ and $\geq 50 \%$ migraine responder rates for the eptinezumab $100 \mathrm{mg}$ and $300 \mathrm{mg}$ dose groups were greater than for placebo. These findings are consistent with previous results from the phase 2 study of $\mathrm{CM}^{22}$

The preventive effects of eptinezumab in patients with $\mathrm{CM}$ were demonstrated on the first day after administration. During the baseline run-in phase, patients experienced a migraine headache on $57.5 \%$ of days. On day 1 after administration, $42.3 \%$ of placebo-treated patients reported having a migraine headache compared with $28.6 \%$ of patients treated with eptinezumab $100 \mathrm{mg}$ and $27.8 \%$ of patients treated with eptinezumab $300 \mathrm{mg}$. This $>50 \%$ reduction in migraine the day after dosing is statistically significant. The reduction in migraine that occurred on day 1 was maintained throughout the study. Over weeks 1 to 4 , migraine occurred in $30.6 \%$ in the eptinezumab $100 \mathrm{mg}$ group, $27.9 \%$ in the eptinezumab $300 \mathrm{mg}$ group, and $38.9 \%$ in the placebo group.

Speed of onset of preventive benefit is viewed by patients as desirable, second only to effectiveness. ${ }^{23}$ Current oral preventive therapies usually require dose titration and develop benefits over weeks to months. ${ }^{24}$ Early onset of effect with eptinezumab may translate into meaningful benefits for patients with CM, allowing them to get back to work, school, household, and family obligations sooner than with previously available options.

Treatment with either dose of eptinezumab was associated with reductions in days of acute medication use. Reduction in acute medication use is an important benefit because overuse of acute medication is associated with headache exacerbation, side effects, and cost. ${ }^{25}$ Reduction in acute medication use has been identified as a positive factor influencing patient acceptance of preventive therapy. ${ }^{26}$ Eptinezumab was well tolerated in this study. The percentages of patients with any TEAEs were similar across the active and placebo groups; most events were mild or moderate in severity, and no deaths were reported. Eptinezumab was similarly well tolerated in previous clinical study reports. ${ }^{22,27}$

The immune response to eptinezumab was characterized by positive ADA results at every time point through week 32 (peak at week 24), with no clear dose-response trend in the number of patients with $\mathrm{ADA}$. The data show a remarkably consistent treatment response to both the 100 and $300 \mathrm{mg}$ doses of eptinezumab, with no impacts on the safety or efficacy profiles of the drug.

PROMISE-2 was designed for consistency with guidelines recently (2018) updated by the International Headache Society Clinical Trials Standing Committee. ${ }^{28}$ It should be noted that individuals with a history or evidence of significant cardiovascular disease or any clinically significant concurrent medical condition were excluded from participation; thus, the findings may not be indicative of safety and efficacy in patients with excluded comorbid conditions. 
Figure 4 Key secondary endpoints (full analysis population)

A. $\geq 75 \%$ migraine response

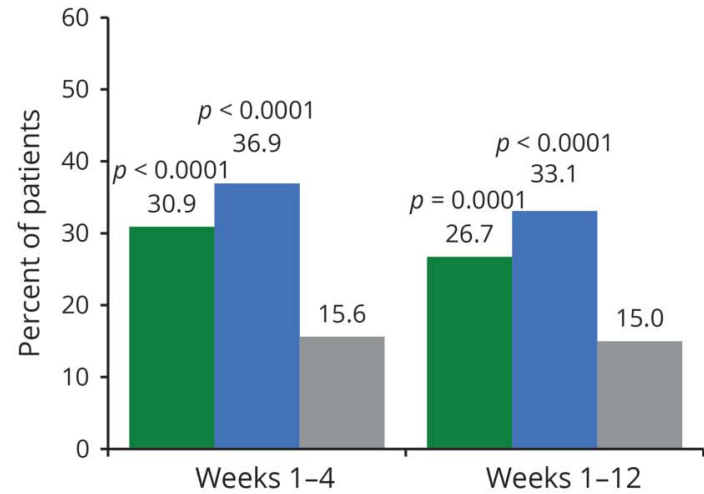

C. Percent of patients with migraine

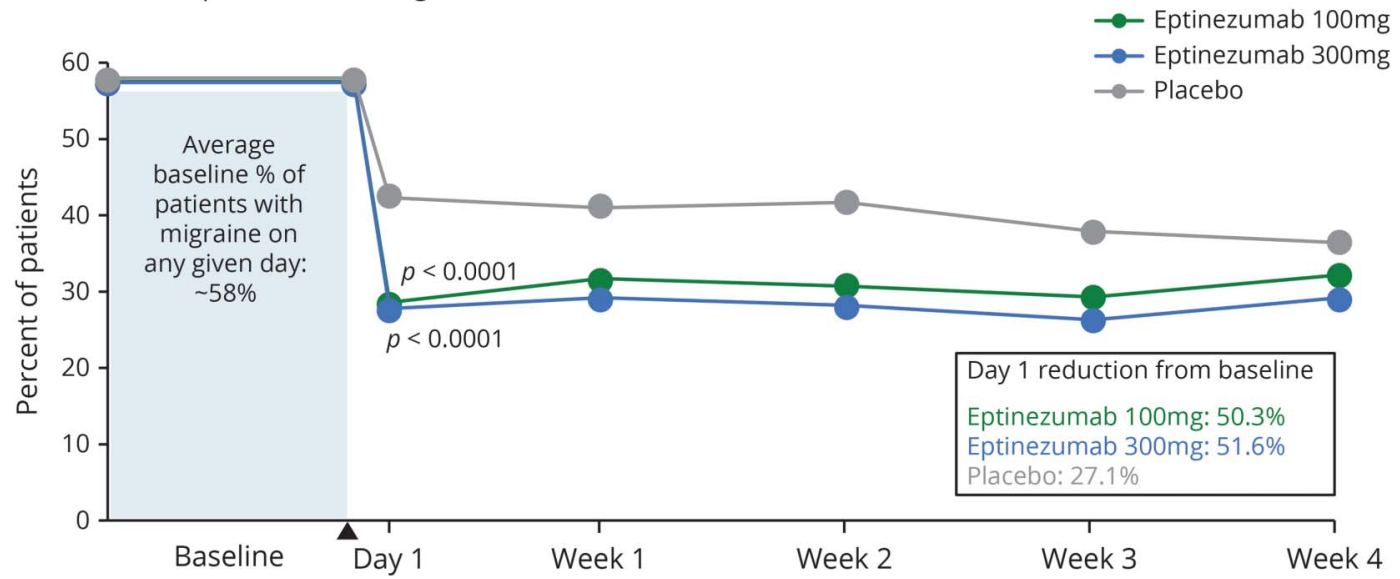

B. $\geq 50 \%$ migraine response

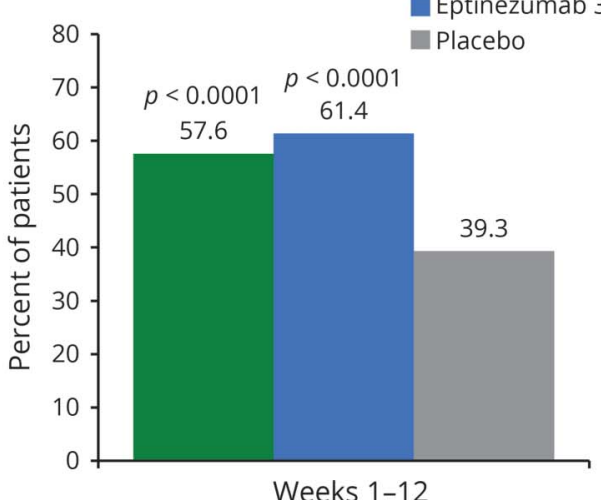

Weeks 1-12
Eptinezumab $100 \mathrm{mg}$ Eptinezumab 300mg Placebo

39.3

(A) $\geq 75 \%$ migraine responder rates, $(B) \geq 50 \%$ migraine responder rates, and (C) patients with migraine on day 1 and during weeks 1 to 4 .

The overall response to placebo was high in this trial. High placebo rates may be attributed to a number of factors, including the novelty of treatment, route of administration, number of active treatment arms increasing patient expectations, and number of patients previously naive to preventive 
Despite the high placebo rates, statistical significance was achieved for eptinezumab $100 \mathrm{mg}$ and $300 \mathrm{mg}$ for the primary and all secondary endpoints.

The results of this study demonstrate that IV eptinezumab (100 and $300 \mathrm{mg}$ ) is associated with a clinically meaningful migraine preventive effect over multiple efficacy measures, is well tolerated, and has an acceptable safety profile in adult patients with $\mathrm{CM}$. The migraine preventive effect of eptinezumab 100 and $300 \mathrm{mg}$ was observed as early as day 1 after administration, with $>50 \%$ of patients reporting a significant decrease in migraine incidence compared to baseline levels. Furthermore, during the first month after the initial administration, more than one-third of patients in the eptinezumab 100 and $300 \mathrm{mg}$ groups experienced a $\geq 75 \%$ reduction in migraine days. Similar meaningful results were reported for clinical and patient-reported outcomes as early as month 1 . The migraine preventive effect of eptinezumab 100 and $300 \mathrm{mg}$ was maintained throughout the full 12 -week dosing interval, with patients who received a single dose of eptinezumab experiencing significantly greater reductions from baseline in mean migraine days during weeks 1 to 12 relative to those receiving placebo.

\section{Acknowledgment}

The authors thank the patients, their families, and the study sites that participated in this study. The authors also thank Mary Tom, PhD, Nicole Coolbaugh, CMPP, and Philip Sjostedt, BPharm, of The Medicine Group, LLC (New Hope, PA) for providing medical writing, editorial, and graphical support, which was funded by $\mathrm{H}$. Lundbeck A/S (Copenhagen, Denmark) in accordance with Good Publication Practice guidelines.

\section{Study funding}

Funded by H. Lundbeck A/S, Copenhagen, Denmark.

\section{Disclosure}

R.B. Lipton reports being a consultant, being an advisory board member, and/or having received honoraria from $\mathrm{Al}$ der BioPharmaceuticals, Allergan, American Academy of Neurology, American Headache Society, Amgen, Autonomic Technologies, Avanir Pharmaceuticals, Biohaven Pharmaceuticals, BioVision, Boston Scientific, Dr. Reddy's Laboratories, ElectroCore Medical, Eli Lilly, eNeura Therapeutics, GlaxoSmithKline, Merck, Pernix, Pfizer, Supernus, Teva Pharmaceuticals, Trigemina, Vector, and Vedanta; reports receiving compensation from eNeura and Biohaven Pharmaceuticals; reports having stock options in Biohaven Pharmaceuticals; and reports research support from Amgen, Migraine Research Foundation, and National Headache Foundation. P.J. Goadsby reports grants and personal fees from Amgen and Eli Lilly and Co; reports personal fees from Alder BioPharmaceuticals, Allergan, Autonomic Technologies, Biohaven Pharmaceuticals, Dr Reddy's Laboratories, Electrocore LLC, eNeura, Impel Neuropharma, MundiPharma, Novartis, Oxford University Press, Teva Pharmaceuticals, Trigemina, Massachusetts Medical Society, Up-to-
Date, and Wolters Kluwer; and reports a patent for magnetic stimulation for headache assigned to eNeura without fee. J. Smith at the time of the study was was a full-time employee of Alder BioPharmaceuticals (currently known as Lundbeck Seattle BioPharmaceuticals). B.A. Schaeffler is a full-time employee of Lundbeck Seattle BioPharmaceuticals. D.M. Biondi at the time of the study was a full-time employee of Alder BioPharmaceuticals (currently known as Lundbeck Seattle BioPharmaceuticals). J. Hirman is a contracted service provider of biostatistical resources to Lundbeck Seattle BioPharmaceuticals. S. Pederson is a full-time employee of Lundbeck Seattle BioPharmaceuticals. B. Allan at the time of the study was a full-time employee of Alder BioPharmaceuticals (currently known as Lundbeck Seattle BioPharmaceuticals). R. Cady is a full-time employee of Lundbeck Seattle BioPharmaceuticals. Go to Neurology. org/ $\mathrm{N}$ for full disclosures.

\section{Publication history}

Received by Neurology April 30, 2019. Accepted in final form August 30, 2019.

\section{Appendix Authors}

\begin{tabular}{|c|c|c|}
\hline Name & Location & Contribution \\
\hline $\begin{array}{l}\text { Richard B. } \\
\text { Lipton, MD }\end{array}$ & $\begin{array}{l}\text { Montefiore Headache } \\
\text { Center; Albert Einstein } \\
\text { College of Medicine, Bronx, } \\
\text { NY }\end{array}$ & $\begin{array}{l}\text { Major role in the } \\
\text { acquisition, analysis, or } \\
\text { interpretation of data; } \\
\text { revised manuscript } \\
\text { critically for important } \\
\text { intellectual content }\end{array}$ \\
\hline $\begin{array}{l}\text { Peter J. } \\
\text { Goadsby, } \\
\text { MD, PhD }\end{array}$ & $\begin{array}{l}\text { NIHR-Wellcome Trust King's } \\
\text { Clinical Research Facility, } \\
\text { King's College London, UK }\end{array}$ & $\begin{array}{l}\text { Major role in the } \\
\text { acquisition, analysis, or } \\
\text { interpretation of data; } \\
\text { revised manuscript } \\
\text { critically for important } \\
\text { intellectual content }\end{array}$ \\
\hline $\begin{array}{l}\text { Jeff Smith, } \\
\text { MD, FRCP }\end{array}$ & $\begin{array}{l}\text { Lundbeck Seattle } \\
\text { BioPharmaceuticals, Inc, } \\
\text { Dublin, Ireland }\end{array}$ & $\begin{array}{l}\text { Major role in the conception } \\
\text { or design of the study; } \\
\text { revised manuscript } \\
\text { critically for important } \\
\text { intellectual content }\end{array}$ \\
\hline
\end{tabular}

\begin{tabular}{ll}
\hline Barbara A. & Lundbeck Seattle \\
Schaeffler, & BioPharmaceuticals, Inc, \\
MBA & Bothell, WA
\end{tabular}

Major role in the acquisition, analysis, or interpretation of data; revised manuscript critically for important intellectual content

\begin{tabular}{ll}
\hline David M. & Cohen Veterans Bioscience, \\
Biondi, DO & Cambridge, MA
\end{tabular}

Major role in the conception and study design and the acquisition, analysis, interpretation of data; revised manuscript critically for important intellectual content

\begin{tabular}{lll}
\hline Joe & Pacific Northwest Statistical & Major role in the \\
Hirman, & Consulting, Inc, & acquisition, analysis, or \\
PhD & Woodinville, WA & interpretation of data; \\
& revised manuscript \\
& critically for important \\
& intellectual content. \\
& Responsible for \\
biostatistical analysis
\end{tabular}


Appendix (continued)

\begin{tabular}{|c|c|c|}
\hline Name & Location & Contribution \\
\hline $\begin{array}{l}\text { Susan } \\
\text { Pederson, } \\
\text { BS }\end{array}$ & $\begin{array}{l}\text { Lundbeck Seattle } \\
\text { BioPharmaceuticals, Inc, } \\
\text { Bothell, WA }\end{array}$ & $\begin{array}{l}\text { Major role in the } \\
\text { acquisition, analysis, or } \\
\text { interpretation of data; } \\
\text { revised manuscript } \\
\text { critically for important } \\
\text { intellectual content }\end{array}$ \\
\hline $\begin{array}{l}\text { Brent } \\
\text { Allan, DO }\end{array}$ & $\begin{array}{l}\text { Global Safety Docs, } \\
\text { Paradise Valley, AZ }\end{array}$ & $\begin{array}{l}\text { Major role in the } \\
\text { acquisition, analysis, or } \\
\text { interpretation of data; } \\
\text { revised manuscript } \\
\text { critically for important } \\
\text { intellectual content }\end{array}$ \\
\hline $\begin{array}{l}\text { Roger } \\
\text { Cady, MD }\end{array}$ & $\begin{array}{l}\text { Lundbeck Seattle } \\
\text { BioPharmaceuticals, Inc, } \\
\text { Bothell, WA }\end{array}$ & $\begin{array}{l}\text { Major role in study design, } \\
\text { the acquisition, analysis, } \\
\text { interpretation of data; } \\
\text { revised manuscript } \\
\text { critically for important } \\
\text { intellectual content }\end{array}$ \\
\hline
\end{tabular}

\section{References}

1. GBD 2016 Headache Collaborators. Global, regional, and national burden of migraine and tension-type headache, 1990-2016: a systematic analysis for the Global Burden of Disease Study 2016. Lancet Neurol 2018;17:954-976.

2. Headache Classification Committee of the International Headache Society (IHS). The International Classification of Headache Disorders, 3rd edition (beta version). Cephalalgia 2013;33:629-808.

3. Adams AM, Serrano D, Buse DC, et al. The impact of chronic migraine: the Chronic Migraine Epidemiology and Outcomes (CaMEO) Study methods and baseline results. Cephalalgia 2015;35:563-578

4. BOTOX (onabotulinumtoxinA) [package Insert]. Madison, NJ: Allergan, Inc; 2018

5. AJOVY [package insert]. North Wales, PA: Teva Pharmaceuticals USA; 2019.

6. AIMOVIG [package insert]. Thousand Oaks, CA: Amgen Inc.; 2019.

7. EMGALITY [package insert]. Indianapolis, IN: Eli Lilly and Company; 2018

8. Lipton RB, Bigal ME, Diamond M, Freitag F, Reed ML, Stewart WF. Migraine prevalence, disease burden, and the need for preventive therapy. Neurology 2007;68: 343-349.

9. Lipton RB, Munjal S, Alam A, et al. Migraine in America Symptoms and Treatment (MAST) Study: baseline study methods, treatment patterns, and gender differences. Headache 2018;58:1408-1426.

10. Hepp Z, Dodick DW, Varon SF, et al. Persistence and switching patterns of oral migraine prophylactic medications among patients with chronic migraine: a retrospective claims analysis. Cephalalgia 2017;37:470-485.

11. Hepp Z, Bloudek LM, Varon SF. Systematic review of migraine prophylaxis adherence and persistence. J Manag Care Spec Pharm 2014;20:22-33.

12. Ramsey RR, Ryan JL, Hershey AD, Powers SW, Aylward BS, Hommel KA. Treatment adherence in patients with headache: a systematic review. Headache 2014;54: 795-816.

13. Berger A, Bloudek LM, Varon SF, Oster G. Adherence with migraine prophylaxis in clinical practice. Pain Pract 2012;12:541-549.
14. Baker B, Schaeffler B, Cady R, Latham J, Whitaker T, Smith J. Rational design of a monoclonal antibody $(\mathrm{mAB})$ inhibiting calcitonin gene-related peptide (CGRP), ALD403, intended for the prevention of migraine (p2.155). Neurology 2017;88.

15. Karasek C, Ojala E, Allison D, Latham J. Characterization of the intrinsic binding features of three anti-CGRP therapeutic antibodies effective in preventing migraine: a comparative pre-clinical case study of ALD403, LY-2951742, TEV-48125. Headache 2016;56.

16. Edvinsson L, Haanes KA, Warfvinge K, Krause DN. CGRP as the target of new migraine therapies: successful translation from bench to clinic. Nat Rev Neurol 2018; 14:338-350.

17. Kosinski M, Bayliss MS, Bjorner JB, et al. A six-item short-form survey for measuring headache impact: the HIT-6. Qual Life Res 2003;12:963-974.

18. Yang M, Rendas-Baum R, Varon SF, Kosinski M. Validation of the Headache Impact Test (HIT-6) across episodic and chronic migraine. Cephalalgia 2011;31:357-367.

19. Posner K, Brent D, Lucas C, et al. Columbia-Suicide Severity Rating Scale (C-SSRS). New York: Columbia University. 2009. Available at: cssrs.columbia.edu/scales practice_cssrs.html. Accessed October 2, 2015.

20. Hintze JL. Power Analysis and Sample Size System (PASS) for Windows user's guide I.Kaysville, UT: NCSS. 2008. Available at: ncss-wpengine.netdna-ssl.com/wp-content/uploads/2012/09/PASSUG1.pdf. Accessed November 19, 2018

21. Holm S. A simple sequentially rejective multiple test procedure. Scand J Stat 1979;6: $65-70$.

22. Goadsby PJ, Smith J, Dodick D., et al. Migraine preventive benefits of ALD403 (eptinezumab) begin in the first 24 hours following intravenous administration. Cephalalgia 2017;37:38.

23. Peres MF, Silberstein S, Moreira F, et al. Patients' preference for migraine preventive therapy. Headache 2007;47:540-545

24. Kumar A, Kadian R. Headache, Migraine Prophylaxis. StatPearls. Treasure Island, FL: StatPearls Publishing; 2018.

25. Suh GI, Park JW, Shin HE. Differences in clinical features and disability according to the frequency of medication use in patients with chronic migraine. J Clin Neurol 2012, 8:198-203.

26. Dekker F, Knuistingh Neven A, Andriesse B, et al. Prophylactic treatment of migraine; the patient's view, a qualitative study. BMC Fam Pract 2012;13:13.

27. Dodick DW, Goadsby PJ, Silberstein SD, et al. Safety and efficacy of ALD403, an antibody to calcitonin gene-related peptide, for the prevention of frequent episodic migraine: a randomised, double-blind, placebo-controlled, exploratory phase 2 trial. Lancet Neurol 2014;13:1100-1107.

28. Tassorelli C, Diener HC, Dodick DW, et al. Guidelines of the International Headache Society for controlled trials of preventive treatment of chronic migraine in adults. Cephalalgia 2018;38:815-832.

29. Couch JR Jr. Placebo effect and clinical trials in migraine therapy. Neuroepidemiology 1987;6:178-185.

30. Pollo A, Amanzio M, Arslanian A, Casadio C, Maggi G, Benedetti F. Response expectancies in placebo analgesia and their clinical relevance. Pain 2001;93:77-84.

31. Antonaci F, Chimento P, Diener HC, Sances G, Bono G. Lessons from placebo effects in migraine treatment. J Headache Pain 2007;8:63-66.

32. de Craen AJ, Tijssen JG, de Gans J, Kleijnen J. Placebo effect in the acute treatment of migraine: subcutaneous placebos are better than oral placebos. J Neurol 2000;247:183-188.

33. Dalakas MC, Rakocevic G, Dambrosia JM, Alexopoulos H, McElroy B. A doubleblind, placebo-controlled study of rituximab in patients with stiff person syndrome. Ann Neurol 2017;82:271-277.

34. Kam-Hansen S, Jakubowski M, Kelley JM, et al. Altered placebo and drug labeling changes the outcome of episodic migraine attacks. Sci Transl Med 2014;6:218ra215.

35. Benedetti F. Placebo effects: from the neurobiological paradigm to translational implications. Neuron 2014;84:623-637. 


\section{Neurology}

Efficacy and safety of eptinezumab in patients with chronic migraine: PROMISE-2

Richard B. Lipton, Peter J. Goadsby, Jeff Smith, et al.

Neurology 2020;94;e1365-e1377 Published Online before print March 24, 2020

DOI 10.1212/WNL.0000000000009169

This information is current as of March 24, 2020

\section{Updated Information \&} Services

References

Subspecialty Collections

Errata

Permissions \& Licensing

Reprints including high resolution figures, can be found at: http://n.neurology.org/content/94/13/e1365.full

This article cites 26 articles, 1 of which you can access for free at: http://n.neurology.org/content/94/13/e1365.full\#ref-list-1

This article, along with others on similar topics, appears in the following collection(s):

All Headache

http://n.neurology.org/cgi/collection/all_headache

Class I

http://n.neurology.org/cgi/collection/class_1

Clinical trials Randomized controlled ( $\bar{C}$ ONSORT agreement)

http://n.neurology.org/cgi/collection/clinical_trials_randomized_contro

lled_consort_agreement

Migraine

http://n.neurology.org/cgi/collection/migraine

An erratum has been published regarding this article. Please see next page or: /content/early/2023/03/24/WNL.0000000000207168.full.pdf

Information about reproducing this article in parts (figures,tables) or in its entirety can be found online at:

http://www.neurology.org/about/about_the_journal\#permissions

Information about ordering reprints can be found online:

http://n.neurology.org/subscribers/advertise

Neurology ${ }^{\circledR}$ is the official journal of the American Academy of Neurology. Published continuously since 1951 , it is now a weekly with 48 issues per year. Copyright Copyright (C) 2020 The Author(s). Published by Wolters Kluwer Health, Inc. on behalf of the American Academy of Neurology.. All rights reserved. Print ISSN: 0028-3878. Online ISSN: 1526-632X.

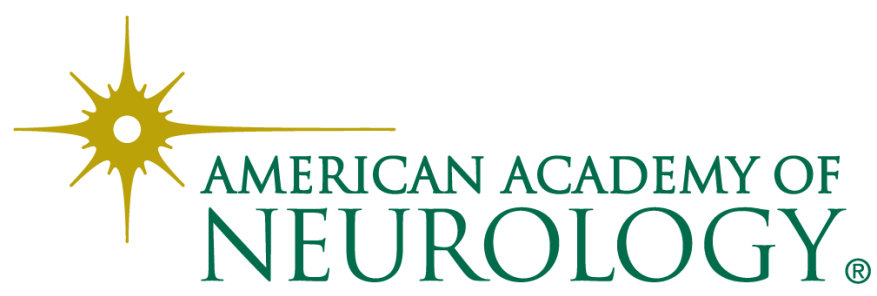




\section{Neurology}

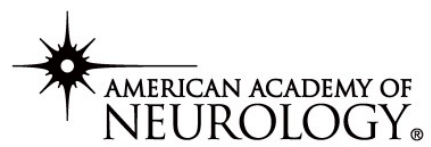

The most widely read and highly cited peer-reviewed neurology journal

The Official Journal of the American Academy of Neurology

\section{Neurology Publish Ahead of Print \\ DOI: 10.1212/WNL.0000000000207168}

\section{CORRECTION \& REPLACEMENT}

\section{Efficacy and Safety of Eptinezumab in Patients With Chronic Migraine: PROMISE-2}

The Research Article "Efficacy and Safety of Eptinezumab in Patients With Chronic Migraine: PROMISE2 " by Lipton et al. ${ }^{1}$, had several minor data errors. The article has been replaced by a corrected version with the link included. The original version with the changes highlighted is available from a link in the corrected article. The authors regret the errors.

\section{REFERENCE}

1 Lipton RB, Goadsby PJ, Smith J, et al. Efficacy and safety of eptinezumab in patients with chronic migraine: PROMISE-2. Neurology. 2020;94(13): e1365-e1377.

Neurology ${ }^{\circledR}$ Published Ahead of Print articles have been peer reviewed and accepted for publication. This manuscript will be published in its final form after copyediting, page composition, and review of proofs. Errors that could affect the content may be corrected during these processes. 